\title{
EduTrail: Um modelo para acompanhamento ubíquo de grupos de aprendizes usando históricos de contextos
}

\author{
Arnóbio F. da Nóbrega ${ }^{1}$, Jorge L. V. Barbosa², João C. Gluz ${ }^{2}$, Luis \\ Gustavo A. Ferreira ${ }^{2}$, Sandro J. Rigo ${ }^{2}$ \\ ${ }^{1}$ Instituto Federal de Educação, Ciência e Tecnologia de Roraima (IFRR) \\ ${ }^{2}$ Universidade do Vale do Rio dos Sinos (UNISINOS) \\ Programa de Pós-Graduação em Computação Aplicada - PPGCA \\ narnobiodifrr.edu.br, \{jbarbosa, rigo\}@unisinos.br, \\ jcgluz@gmail.com, luis.gustavo.af@gmail.com
}

\begin{abstract}
This article proposes a model for automated recording of contexts histories of learners' groups, called EduTrail. The model presents a muti-agent architecture using the Prometheus methodology. In addition, the text shows the implementation aspects of each agent. A scenario was applied to evaluate the EduTrail, involving 3 pedagogues, 3 teachers and 41 students. At the end of the scenario, a questionnaire based on TAM methodology was applied to the participants. The pedagogues indicated 66\% of "Excelent" to perceived ease of use and $100 \%$ in perceived usefulness.
\end{abstract}

Resumo: Este artigo propõe um modelo para registro automatizado de históricos de contextos de grupos de aprendizes, denominado EduTrail. O modelo apresenta uma arquitetura muti-agente utilizando a metodologia Prometheus. Além disso, o texto descreve os aspectos de implementação de cada agente. Um cenário foi aplicado na avaliação do EduTrail, envolvendo 3 pedagogos, 3 professores e 41 alunos. Após a execução do cenário, um questionário baseado na metodologia TAM foi aplicado aos participantes. Os pedagogos indicaram $66 \%$ de "Excelente" para a facilidade de uso percebida e $100 \%$ para a utilidade percebida.

\section{Introdução}

A produção e a difusão crescente de novas Tecnologias Digitais de Comunicação e Informação (TDCI) impacta a gestão de ambientes organizacionais, requerendo dos gestores mais agilidade, flexibilidade, inovação e capacidade de adaptação [Carvalho et al. 2018]. Uma alternativa para o acompanhamento pedagógico em instituições de ensino é o desenvolvimento de modelos que permitam o registro de históricos de contextos de aprendizagem. Esses modelos levam em conta as informações 
provenientes dos temas e tópicos dos planos de ensino, dos perfis individuais dos aprendizes e dos grupos de aprendizes. Além disso, a computação ubíqua [Cárdenas-Robledo e Peña-Ayala 2018] vem estimulando o desenvolvimento de sistemas nos quais o contexto dos usuários é levado em consideração na disponibilização de serviços.

Apesar de um existir um número grande de trabalhos dedicados à aplicação de contextos para apoiar as necessidades de alunos, não observase o mesmo empenho no desenvolvimento de recursos voltados às necessidades dos professores. Visando atuar nesta lacuna, este artigo propõe o modelo EduTrail para registro e acompanhamento dos históricos de contextos [Rosa et al. 2015] de grupos de aprendizes. Destaca-se como contribuição científica o uso de históricos de contextos para auxílio aos professores e pedagogos e a sua avaliação crítica em um experimento controlado.

$\mathrm{O}$ artigo está organizado em sete seções. A seção 2 discute os trabalhos relacionados. A seção 3 descreve o modelo EduTrail. A seção 4 aborda aspectos relacionados à implementação. A seção 5 apresenta um estudo de caso. A seção 6 discute os resultados do experimento. Na seção 7 encontram-se as considerações finais.

\section{Trabalhos Relacionados}

Os seguintes critérios foram considerados estratégicos tanto para escolha dos trabalhos relacionados quanto para comparação entre eles: a) Agentes de Software: identifica o uso de tecnologia de agentes de software conforme definição de [Silveira e Barone 2006]; b) Sensível ao Contexto: identifica se foi utilizada alguma informação de contexto [Dey et al. 2001]; c) Dispositivos Móveis: identifica o uso de dispositivos móveis; d) Técnicas de recomendação: identifica se foi utilizada alguma técnica de recomendação; e) Perfis: identifica se o trabalho utiliza perfis de aprendiz, segundo [Levis et al. 2008]; f) Histórico de Contextos: identifica se o trabalho registra e usa históricos de contextos [Rosa et al. 2015].

Silva et al. (2010) [Silva et al. 2010] propuseram o UbiTrail para o gerenciamento de trilhas e sua aplicação na distribuição de conteúdo. Embora o UbiTrail tenha sido integrado a um sistema de educação ubíqua denominado LOCAL, sua proposta é genérica o bastante para ser aplicada em outros sistemas que possam usufruir de históricos de contextos. O trabalho apresentado em [Levis et al. 2008] é chamado de PeLep. O modelo propõe o uso de perfis aperfeiçoados automaticamente em ambientes de educação ubíqua. [Silveira e Barone 2006] propuseram a formação de grupos colaborativos a partir de critérios que podem ser configurados pelo professor. O estudo apresentado por [Ferreira et al. 2015] propõe um modelo chamado UbiGroup, o qual realiza recomendação ubíqua de conteúdo para grupos de aprendizes. O UbiGroup recomenda materiais educacionais considerando de forma conjunta os perfis de um grupo de aprendizes e o contexto de ensino no qual eles se encontram. 
A Tabela 1 evidencia a contribuição do EduTrail na estratégia para registro e gerenciamento de históricos de contextos de ensino dos aprendizes, gerando subsídios para apoio aos professores e gestores.

Tabela 1: Comparativo entre os Trabalhos Relacionados

\begin{tabular}{|c|c|c|c|c|c|c|}
\hline Trabalho & Agente de Software & $\begin{array}{c}\text { Sensível ao } \\
\text { Contexto }\end{array}$ & $\begin{array}{c}\text { Dispositivos } \\
\text { Móveis }\end{array}$ & $\begin{array}{c}\text { Técnicas para } \\
\text { Recomendação }\end{array}$ & $\begin{array}{c}\text { Perfis } \\
\text { Histórico } \\
\text { de } \\
\text { Contextos }\end{array}$ \\
\hline UbiTrail/LOCAL & SIM & SIM & SIM & NÃO & SIM & NÃO \\
\hline PeLep & SIM & SIM & SIM & NÃO & SIM & NÃO \\
\hline Projeto AMIA & SIM & NÃO & NÃO & NÃO & NÃO & NÃO \\
\hline UbiGroup & SIM & SIM & SIM & SIM & SIM & NÃO \\
\hline EduTrail & SIM & SIM & SIM & SIM & SIM & SIM \\
\hline
\end{tabular}

\section{Modelo Edutrail}

O EduTrail registra históricos dos contextos de aprendizes, a partir de informações provenientes dos temas e tópicos do plano de ensino, bem como da entrada e saída dos alunos nos contextos de ensino. Para tal, o EduTrail foi integrado ao modelo UbiGroup [Ferreira et al. 2015], em uma arquitetura composta por agentes de software, apresentada na Figura 1.

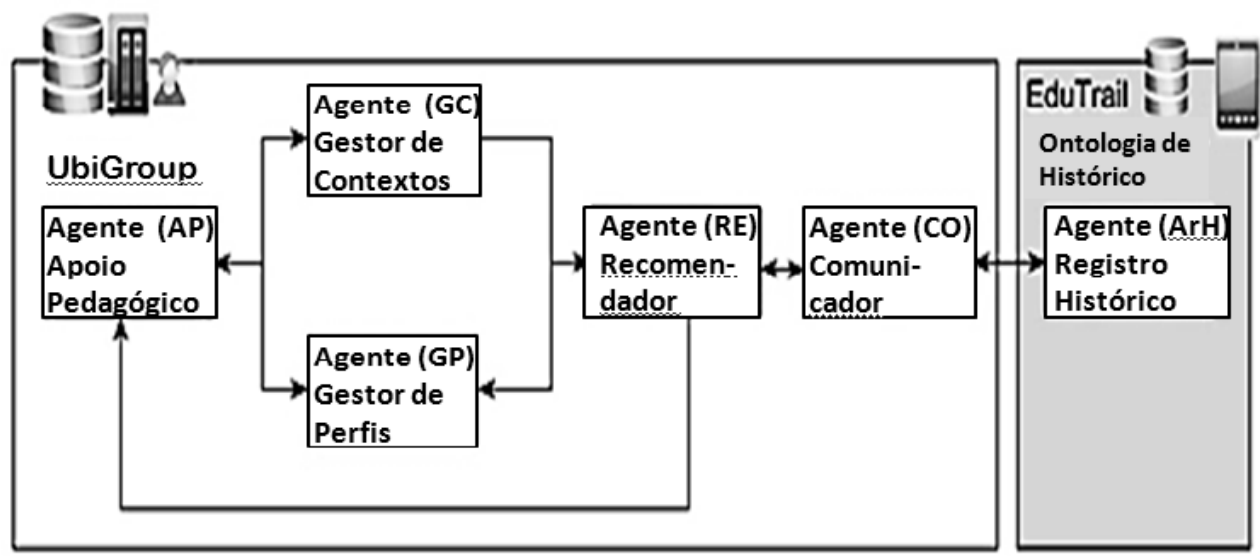

Figura 1. Arquitetura do EduTrail integrado ao UbiGroup.

\subsection{Agentes de Software}

Os agentes de softwares definidos no modelo UbiGroup são os seguintes. Agente Gestor de Contexto (GC): agente responsável por monitorar as atividades dos aprendizes e recuperar da ontologia de contexto as pontuações de aprendizagem que compõem os perfis dos aprendizes e os seus históricos de acesso aos Objetos de Aprendizagem (OAs). Agente Recomendador (RE): agente responsável por detectar OAs que podem ser recomendados, conforme o contexto do aprendiz, de acordo com as informações fornecidas pelo GC e as informações obtidas dos OAs. O agente RE gerencia o processo de recomendação. Agente Pedagógico (AP): agente responsável por realizar a interface com o aprendiz. O agente AP envia para o agente GP as informações atualizadas dos perfis e repassa a posição atualizada para o agente GC. Agente Comunicador (CO): agente responsável pela comunicação com os repositórios de OAs. A partir de uma solicitação do agente ArH (descrito na próxima subseção), ele efetua o acesso ao repositório de históricos de contextos e retorna as informações 
solicitadas. Agente Gestor de Perfis (GP): agente responsável pelo registro de desempenho, isco de evasão e ou retenção, sendo também responsável por manter os perfis dos aprendizes atualizados e por gerar similaridades entre os perfis que estão no contexto.

\subsection{Agente de Registro de Históricos}

$\mathrm{O}$ agente Registro de Históricos (ArH) registra os históricos dos contextos de aprendizes a partir das informações mantidas pelo Agente de Contexto (GC) durante as alterações ocorridas com relação à entrada e a saída de aprendizes dos contextos. A movimentação é monitorada pelo agente AP, o qual envia esta informação ao agente GC. A Figura 2 mostra o agente ArH modelado usando a metodologia Prometheus. Suas capacidades estão definidas como: a) Criar Histórico dos Contextos: capacidade de armazenar informações da movimentação dos aprendizes; b) Enviar informações de Entrada e Saída do Aprendiz: capacidade de disponibilizar informações do registro de histórico dos contextos; c) Receber informações das entradas e saídas do aprendiz: capacidade de receber informações do registro do histórico dos contextos fazendo a notificação do movimento do aprendiz; d) Listar os registros de Histórico dos Contextos: capacidade de gerar listagem dos registros do Histórico dos Contextos.

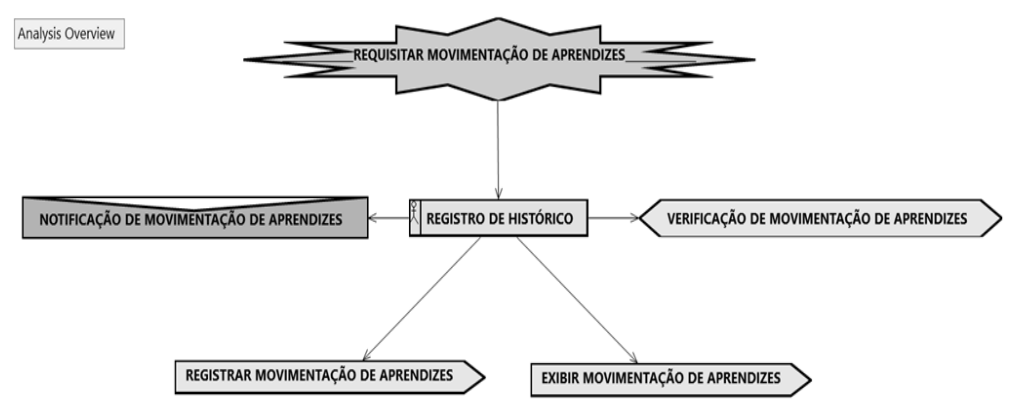

Figura 2. Agente Registro de Históricos (ArH)

Tendo como base as definições de [Dey et al. 2001] foi proposta uma ontologia para tratamento dos históricos de contextos, chamada OntoHC (Figura 3). Essa ontologia auxilia os agentes nas trocas de mensagens, mantendo um padrão no tratamento das informações.

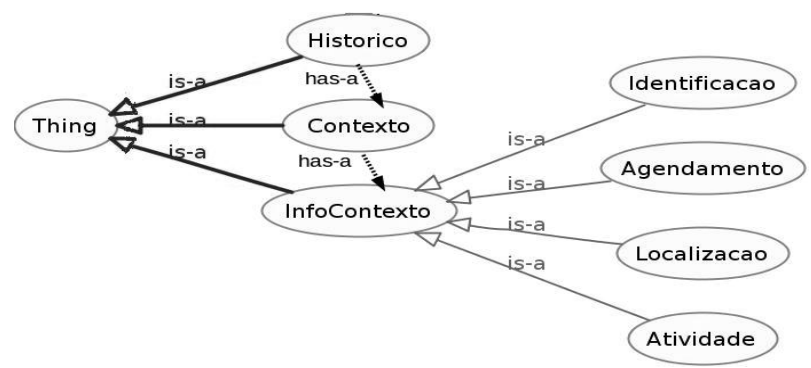

Figura 3. Ontologia Históricos dos Contextos (OntoHC) 
A OntoHC define os termos do domínio de movimentação dos aprendizes. A seguir são detalhadas as classes da ontologia:

- Histórico: classe responsável por registrar a movimentação de aprendizes nos contextos;

- Contexto: classe que identifica o contexto de ensino na qual os aprendizes estão inseridos;

- InfoContexto: classe que define quais informações são registradas em cada contexto;

- Identificação: essa subclasse contém informações que identificam o contexto;

- Atividade: contém informações referentes às atividades que ocorrem no contexto;

- Localização: essa subclasse contém informações sobre o posicionamento geográfico do contexto;

- Agendamento: essa subclasse contém informações referentes ao controle das atividades nos contextos.

\section{Aspectos de Implementação}

A implementação do protótipo seguiu o padrão arquitetônico Model-ViewController (MVC). No desenvolvimento foi utilizada a linguagem de programação Java, um servidor de aplicação, um Kit de desenvolvimento de aplicações para o sistema operacional Android e bibliotecas para auxiliar na comunicação de dados formando assim um ambiente envolvendo: Tomcat, Android, Jena, Framewok Jersey 2.0 e MySql.

\section{Estudo de Caso}

A avaliação do EduTrail foi feita através de um estudo de caso realizado em uma instituição de ensino de cursos técnicos profissionais localizada na região Norte do Brasil. O estudo foi baseado em um levantamento dos requisitos para a implantação da gestão pedagógica voltada para o acompanhamento de um grupo de alunos e com suporte de históricos de contextos. O estudo foi subsidiado por entrevistas com servidores do Departamento Pedagógico.

Um cenário foi criado para avaliação correspondente a uma aula utilizando o EduTrail. Para o estudo foi considerada a seguinte situação: "O Departamento Pedagógico gostaria que fossem registrados os históricos dos contextos durante a aula ministrada pelo professor do ensino médio enquanto os aprendizes presentes na sala de aula recebessem uma recomendação de material educacional no período das $09 \mathrm{~h} 40 \mathrm{~min}$ até 10h40min em seus smartphones". Para a preparação do teste, as informações do contex to foram cadastradas como mostra a Tabela 2. 
Tabela 2: Informações do Contexto

\begin{tabular}{|c|c|c|}
\hline \multicolumn{3}{|c|}{ Contexto Sala 305 IFRR } \\
\hline \multirow{3}{*}{ Localização } & Nome & IFRR \\
\hline & PosicaoGPSIni & 2.8130295 \\
\hline & PosicaoGPSFim & -60.6939089 \\
\hline \multirow{5}{*}{ Atividade } & Tipo & Aula \\
\hline & Nome & Algo. Lógica \\
\hline & Descrição & Aula Lógica Programação \\
\hline & RegraRecomendacao & Regra padrão aula \\
\hline & PalabraChave & Algoritmo \\
\hline \multirow{4}{*}{ Agendamento } & Dia da Semana & Quarta-feira \\
\hline & Data & $21-10-2015$ \\
\hline & HoraInicio & $09: 40$ \\
\hline & HoraFim & $10: 40$ \\
\hline \multirow{2}{*}{ Identificação } & TipoGrupo & Misto \\
\hline & NumeroMinimoAprendizes & 3 \\
\hline
\end{tabular}

O cenário simulou uma situação comum na instituição de ensino. Após o horário do intervalo, os alunos se dirigem a sala de aula, sendo identificados no contexto. Neste momento o grupo de alunos passa a ser acompanhado pelo EduTrail. Ao perceber que um aluno entra no contexto, o agente GP deve atualizar a similaridade de todos os perfis dos aprendizes (Tabela 3 e 4) e enviar as credenciais de acesso do aluno ao agente ArH. Após o agente GP atualizar a similaridade, o agente GC realiza uma verificação quanto ao número mínimo de alunos no contexto, avaliando se foi atingido o número conforme o grau de similaridade. Caso tenha sido atingido, o agente $\mathrm{RE}$ recebe o contexto dos alunos e identifica o perfil mais similar ao grupo (Aluno J, ver Tabela 3). A partir da regra de recomendação contida no contexto, são definidos os parâmetros de consulta aos metadados dos OAs (mostrados na Tabela 5).

Tabela 3: Perfis mais similares no Grupo

\begin{tabular}{|l|c|c|c|c|}
\hline & Aluno: J & Aluno: F & Aluno: G & Prof: V \\
\hline Aluno: J & - & $66 \%$ & $46 \%$ & $52 \%$ \\
\hline Aluno: F & $66 \%$ & - & $42 \%$ & $26 \%$ \\
\hline Aluno: G & $46 \%$ & $46 \%$ & - & $6 \%$ \\
\hline Professor: V & $52 \%$ & $26 \%$ & $6 \%$ & - \\
\hline Média Individual & $41 \%$ & $35 \%$ & $24 \%$ & $21 \%$ \\
\hline
\end{tabular}

Usando regras de recomendação previamente configuradas que fornecem a associação entre o perfil do grupo de alunos e o contexto, o agente RE localiza os OAs mais adequados as estas informações. Logo após, as informações são enviadas para o agente $\mathrm{CO}$, que monta uma consulta SPARQL, usada para extrair o conjunto de OAs que podem ser recomendados da base de OAs. O resultado da consulta é encaminhado para o agente RE que ordena, seleciona e prepara a recomendação a ser encaminhada aos alunos. $O$ agente RE envia essa recomendação do conteúdo para o agente AP e este agente envia uma notificação ao dispositivo móvel. Os alunos visualizam as recomendações e são convidados a responderem se gostaram ou não do material. As indicações realizadas pelos alunos são armazenadas para utilização do agente Recomendador.

$\mathrm{O}$ agente $\mathrm{ArH}$ registra históricos de contextos a partir das informações mantidas pelo agente GC durante as alterações ocorridas com relação à entrada e a saída de membros de algum contexto. No cenário de avaliação, 
VIII Congresso Brasileiro de Informática na Educação (CBIE 2019)

Anais do XXX Simpósio Brasileiro de Informática na Educação (SBIE 2019)

após o encerramento da aula, uma listagem com os dados de acompanhamento dos aprendizes (Tabela 6), foi obtida junto ao Departamento Pedagógico.

O cenário de avaliação foi implementado na sala de aula com a presença de três pedagogos, três professores e quarenta e um alunos. Os participantes receberam instruções detalhadas antes dos procedimentos. Um professor operando o sistema, após fazer o cadastramento da Tabela 2, realizou o direcionamento necessário aos aprendizes presentes na sala de aula. Os alunos foram convidados pelo professor para fazerem seu acesso usando dispositivos móveis (smartphone, tablet ou notebook).

Tabela 4: Perfis do Contexto

\begin{tabular}{|c|c|c|c|c|c|}
\hline \multirow{3}{*}{ ฮี } & IdAprendiz & 01 & 02 & 03 & 04 \\
\hline & Nome & Aluno J & Aluno F & Aluno $\mathrm{G}$ & Professor V \\
\hline & Tipo & Aluno & Aluno & Aluno & Professor \\
\hline \multirow{7}{*}{ 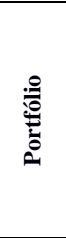 } & IdAprendiz & 01 & 02 & 03 & 04 \\
\hline & TipoPortfolio & Apresentação & Vídeo & Vídeo & Apresentação \\
\hline & Título & $\begin{array}{l}\text { A série de } \\
\text { Fibonacci }\end{array}$ & Tabela Lógica F e V & $\begin{array}{c}\text { Tabela Lógica F e } \\
\text { V }\end{array}$ & A série de Fibonacci \\
\hline & Descricao & & & & \\
\hline & Evento & RNIFRR & RNIFRR & RNIFRR & RNIFRR \\
\hline & Local & Boa Vista & Boa Vista & Boa Vista & Boa Vista \\
\hline & Ano & 2015 & 2015 & 2015 & 2015 \\
\hline \multirow{7}{*}{ 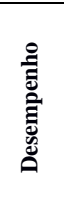 } & IdAprendiz & 01 & 02 & 03 & 04 \\
\hline & TipoDesempenho & Disciplina & Disciplina & Disciplina & - \\
\hline & Nome & Algoritmo & Algoritmo & Algoritmo & - \\
\hline & Descritivo & & & & - \\
\hline & Instituicao & IFRR & IFRR & IFRR & - \\
\hline & Avaliacao & 8,5 & 7,5 & 8,0 & - \\
\hline & DataReferencia & 2015-08 & 2015-08 & 2015-08 & - \\
\hline \multirow{5}{*}{ 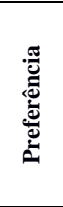 } & IdAprendiz & 01 & 02 & 03 & 04 \\
\hline & FormatoOA & $\begin{array}{l}\text { Vídeo, Audio, } \\
\text { PDF }\end{array}$ & Vídeo, Audio, PDF & $\begin{array}{c}\text { Vídeo, Audio, } \\
\text { PDF }\end{array}$ & Vídeo, Audio, PDF \\
\hline & TipoDispositivo & Mobile & Moble & Mobile & Tablet \\
\hline & Visual & Visual & Visual & Visual & Visual \\
\hline & Contexto & $\begin{array}{c}\text { Campus BV Sala } \\
305\end{array}$ & Campus BV Sala 305 & $\begin{array}{c}\text { Campus BV Sala } \\
305 \\
\end{array}$ & Campus BV Sala 305 \\
\hline
\end{tabular}

Tabela 5: Contexto, Perfis do Aprendiz e Banco de Dados de OAs.

\begin{tabular}{|c|c|c|c|c|c|}
\hline \multirow{8}{*}{ 胥 } & \multicolumn{2}{|c|}{ Contexto } & \multicolumn{3}{|c|}{ Metadados OA (Banco de Dados) } \\
\hline & Atividade & PalavrasChave & General & Title & Algoritmo \\
\hline & Atividade & PalavrasChave & General & Description & Algoritmo \\
\hline & Atividade & Palavraschave & General & Keyword & Algoritmo \\
\hline & \multicolumn{2}{|c|}{ Perfil do Grupo de Aprendizes } & \multicolumn{3}{|c|}{ Metadados AO (Banco de Dados) } \\
\hline & Contato & Tipo & Educacional & IntendedEndUserRolels & Learner \\
\hline & Preferencia & FormatoOA & Segmentation & SegmentMédiaTypels & Video \\
\hline & Preferencia & TipoDispositivo & Technical & SupportedplatformsIs & Mobile \\
\hline
\end{tabular}

Tabela 6: Registro do Histórico dos Contextos

\begin{tabular}{|l|l|l|l|l|l|l|}
\hline \multicolumn{6}{|l}{ Listagem - Registro Histórico dos Contextos } & Data: 30/10/2015 \\
\hline id & Aprendiz & atividade & local & data & hora entrada & hora saída \\
\hline 001 & J & Aula Algoritmo & Sala 305 & $21-10-2015$ & $09: 38$ & \\
\hline 001 & J & Aula Algoritmo & Sala 305 & $21-10-2015$ & & $10: 41$ \\
\hline 002 & F & Aula Algoritmo & Sala 305 & $21-10-2015$ & $09: 41$ & \\
\hline 002 & F & Aula. Algoritmo & Sala 305 & & & $10: 44$ \\
\hline 003 & G & Aula Algoritmo & Sala 305 & $21-10-2015$ & $09: 39$ & \\
\hline 003 & G & Aula Algoritmo & Sala 305 & $21-10-2015$ & & $10: 45$ \\
\hline
\end{tabular}

Ao fim do intervalo os alunos dirigiram-se a sala de aula e, ao atingirem as limitações da posição física do contexto, foram notificados 
através da aplicação. Além da localização, a aplicação verifica as informações de data e hora, identificando automaticamente os alunos que estão vinculados ao contexto. Neste procedimento também é gerada a similaridade entre eles com o objetivo de identificar o perfil mais representativo para o grupo.

No cenário de avaliação, entraram no contexto os três primeiros alunos. A partir de então foi executado o processo de similaridade. A Figura 4 apresenta a interface do grupo de alunos, bem como a similaridade entre eles, ocorrendo o registro de histórico dos contextos desta movimentação.

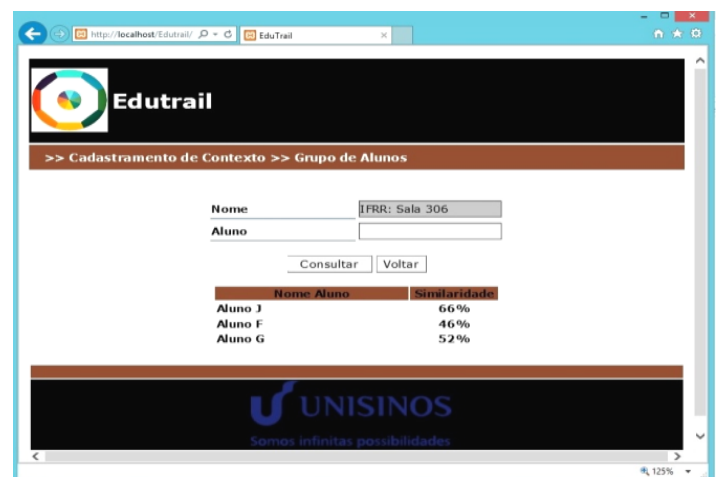

Figura 4. Alunos contidos no contexto.

Como a entrada de três alunos atinge o número mínimo de alunos para o contexto, então o processo de recomendação é executado pela aplicação. O procedimento seguinte consiste em solicitar ao professor a efetivação da recomendação, sendo que este pode aguardar mais alguns minutos, caso identifique que estão faltando muitos alunos. Quando o horário determinado pelo professor (Figura 5, item "a") é atingido, o professor confirma e o processo de recomendação é iniciado.

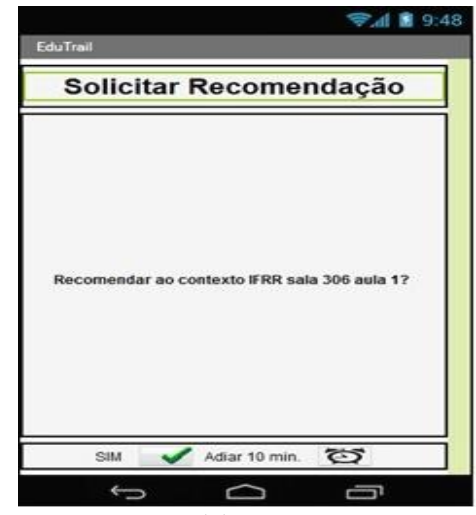

(a)

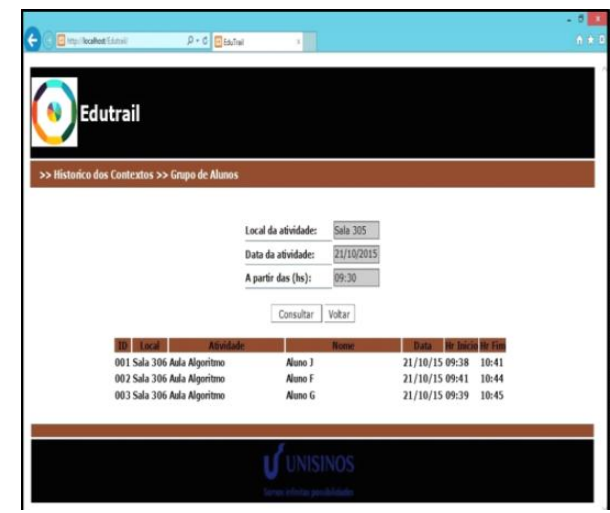

(b)

Figura 5. Alunos no contexto e Consulta Registro Histórico de Contextos

Após o encerramento da aula, uma listagem com os dados de acompanhamento dos aprendizes (Figura 5, item "b") foi gerada para consulta no Departamento Pedagógico. Foi distribuído para o grupo de três pedagogos um questionário de avaliação do experimento contendo 
afirmações conforme a Tabela 7. O questionário foi elaborado para avaliar o desempenho e a relevância da aceitação do EduTrail por parte dos profissionais do Departamento Pedagógico. As respostas foram padronizadas na escala Likert de cinco pontos, variando entre 1 (péssimo) até 5 (excelente). Os itens do questionário foram elaborados com base nos conceitos do modelo de aceitação de tecnologia (TAM - Technology Acceptance Model) [Marangunié and Granié, 2014]. O TAM avalia a aceitação através de dois aspectos: a) Facilidade de uso percebida: grau em que uma pessoa acredita que o uso de um sistema de informação será livre de esforço; b) Utilidade percebida: grau em que uma pessoa acredita que o uso de um sistema pode melhorar o seu desempenho.

\section{Tabela 7: Questionário de Avaliação}

\begin{tabular}{|c|c|}
\hline \multicolumn{2}{|r|}{ Informe sua opinião sobre as seguintes afirmações: } \\
\hline 1 & $\begin{array}{l}\text { Aprender a usar o EduTrail foi fácil? } \\
\text { () Péssimo ( ) Ruim ( ) Bom ( ) Ótimo () Excelente }\end{array}$ \\
\hline 2 & $\begin{array}{l}\text { Comparando o EduTrail com outros instrumentos já utilizados para acompanhamento de aprendizes, ele é? } \\
\text { () Péssimo ( ) Ruim ( ) Bom ( ) Ótimo ( ) Excelente }\end{array}$ \\
\hline 3 & $\begin{array}{l}\text { O uso do EduTrail facilita o trabalho do professor na tarefa de acompanhamento de aprendizes? } \\
\text { () Péssimo ( ) Ruim ( ) Bom ( ) Ótimo ( ) Excelente }\end{array}$ \\
\hline 4 & $\begin{array}{l}\text { O EduTrail facilita a realização do processo de acompanhamento de grupo de aprendizes? } \\
\text { () Péssimo ( ) Ruim ( ) Bom ( ) Ótimo ( ) Excelente }\end{array}$ \\
\hline 5 & $\begin{array}{l}\text { O Relatório Registro de Histórico gerado pelo sistema é útil no processo acompanhamento de aprendizes? } \\
\text { () Péssimo ( ) Ruim ( ) Bom ( ) Ótimo ( ) Excelente }\end{array}$ \\
\hline 6 & $\begin{array}{l}\text { Usar o EduTrail aumentou meu desempenho no processo de resolver suas tarefas? } \\
\text { () Péssimo ( ) Ruim ( ) Bom ( ) Ótimo ( ) Excelente }\end{array}$ \\
\hline 7 & $\begin{array}{l}\text { Você recomendaria a usar o EduTrail como instrumento de prevenção de risco de evasão escolar? } \\
\text { () Péssimo ( ) Ruim ( ) Bom ( ) Ótimo ( ) Excelente }\end{array}$ \\
\hline 8 & $\begin{array}{l}\text { O relatório histórico dos contextos gerado pelo EduTrail durante o acompanhamento de aprendiz em sala de aula satisfaz? } \\
\text { () Péssimo () Ruim ( ) Bom () Ótimo ( ) Excelente }\end{array}$ \\
\hline 9 & $\begin{array}{l}\text { Utilizar o EduTrail é importante e adiciona valor ao processo acompanhamento de aprendizes em sala de aula? } \\
\text { () Péssimo ( ) Ruim ( ) Bom ( ) Ótimo ( ) Excelente ( ) }\end{array}$ \\
\hline 10 & $\begin{array}{l}\text { Usar o EduTrail minimizou o tempo de resposta na realização da atividade acompanhamento de aprendizes? } \\
\text { ( ) Péssimo ( ) Ruim ( ) Bom ( ) Ótimo ( ) Excelente }\end{array}$ \\
\hline
\end{tabular}

\section{Resultados}

Os percentuais obtidos nas afirmações de 1 a 5 respondidas pelo grupo de pedagogos, avaliaram o aspecto relacionado à facilidade de uso percebida. De acordo com as opiniões do grupo entrevistado o grau de dificuldade foi baixo. Mais de 33\% das respostas dos pedagogos foi "Ótimo" em relação às questões 1 à 5, fornecendo boas evidências da facilidade e satisfação com que uma pessoa pode empregar o EduTrail. Mais de $66 \%$ das opiniões do grupo de pedagogos foram "Excelente" para esse conjunto de questões, mostrando uma aceitação do grupo para este processo.

Ainda respondidas pelo grupo de pedagogos, as questões 6 a 10 avaliaram a utilidade percebida do EduTrail. Nessas questões, $100 \%$ das questões foram respondidas com "Excelente", indicando a utilidade do EduTrail como uma ferramenta para acompanhamento de grupos de aprendizes.

\section{Considerações Finais}

Este artigo apresentou o EduTrail, um modelo para gerenciamento dos históricos de contextos educacionais. No cenário de avaliação que simulou uma aula, o EduTrail registrou o histórico dos contextos enquanto ocorria a movimentação dos alunos, entrando e saindo da sala. Além disso, os alunos receberam uma recomendação de material educacional em seus 
smartphones. Os resultados apontam a viabilidade do modelo, além de indicar que o EduTrail é um instrumento de fácil utilização. Ele teve uma boa aceitação quanto ao auxílio nas tarefas realizadas pelo pessoal do Departamento Pedagógico, no acompanhamento de aprendizes através do registro dos históricos dos contextos. A aplicação foi desenvolvida para fazer o tratamento em apenas um contexto na mesma localização física. Se dentro deste contexto existir a intersecção com outro contexto, a aplicação atualmente não realiza nenhuma ação. Esta é uma situação que inspira trabalhos futuros, pelo fato de existirem contextos com maior grau de relevância do que outros.

\section{Agradecimentos}

Os autores agradecem à Fundação de Amparo à Pesquisa do Estado do Rio Grande do Sul, ao Conselho Nacional de Desenvolvimento Científico e Tecnológico, à Universidade do Vale do Rio dos Sinos e ao Instituto Federal de Educação, Ciência e Tecnologia de Roraima.

\section{Referências}

Cárdenas-Robledo, L. A., Peña-Ayala, A. (2018). Ubiquitous learning: A systematic review. Telematics and Informatics, 35(5):1097-1132.

Carvalho, Daiane Aparecida Costa; De Lima, Marcio Roberto. (2018). Formação de professores para o uso pedagógico das tecnologias digitais de informação e comunicação. CIET: EnPED.

Ferreira, L. G. A., et al. (2015). Ubigroup: Um modelo de recomendação ubíqua de conteúdo para grupos dinâmicos de aprendizes. Revista Brasileira de Informática na Educação, 23(3):40.

Gutierrez, C. (2009). Advanced Position Based Services to Improve Accessibility. In: 3rd Symposium of Ubiquitous Computing and Ambient Intelligence 2008. Springer Berlin Heidelberg. Pages 284-292.

Levis, D., et al. (2008). Aperfeiçoamento automático do perfil do aprendiz em ambiente de educação ubíqua. Revista Brasileira de Informática na Educação, 16(1).

Marangunić, N., Granić, A.: Technology acceptance model: aliterature review from 1986 to 2013; Universal Access in the Information Society, 14(1): 81-95.

Rosa, J.H., et al. (2015) A Multi-Temporal Context-Aware System for Competences Management. International Journal of Artificial Intelligence in Education, 25: 455-492.

Silva, J., et al. (2010). Content distribution in trail-aware environments. Journal of the Brazilian Computer Society (Impresso), 16(2):163-176.

Silveira, R. S., and Barone. D. A. C. (2006). Formação de grupos colaborativos em cursos a distância via web: Um estudo de caso utilizando técnicas de inteligência artificial. Revista Brasileira de Informática na Educação. Florianópolis. 14(2):29-40. 